\title{
Risk Analysis of Historic Urban Areas: A Case Study of the Salerno City, Italy
}

\author{
Federica Ribera ${ }^{1}$, Luigi Petti ${ }^{1}$, Gennaro Miccio ${ }^{2}$, Alessandra Landi $^{1}$ and Alessio Lodato ${ }^{1}$ \\ 1. Department of Civil Engineering, University of Salerno, Fisciano 84084, Italy \\ 2. Ministry of Cultural Heritage and Activities and Tourism, Roma 00186, Italy
}

\begin{abstract}
The preservation of historical and cultural heritage from seismic risk is a question of interest in Italy. However, too little has been done to deal with this problem. First of all, the seismic safety of cultural heritage should be a topic of prevention. The research described in the paper presents a multi-approach procedure to address risk assessment of large-scale urban systems with the aim to contribute at the development of risk mitigation policies for historical centers buildings. The observation of the earthquakes effects in Italy clearly highlights the role of the high vulnerability of the existing building on the consequences in terms of casualties and damage, rather than the severity of seismic events, especially in historical contexts. Unlike the new buildings which should be planned and designed to respect seismic safety requirements, existing buildings require a careful safety assessment on the basis of their susceptibility to damage which depends on building techniques, past damage events, maintenance, changes, etc.
\end{abstract}

Key words: Seismic hazard, historical centers, vulnerability.

\section{Introduction}

The purpose of this study is to assess the seismic risk of an urban area in the city of Salerno, characterized by a homogeneous historical buildings, datable to the first half of the 20th century. The preservation of this architectural heritage is essential for its symbolic and cultural value, and for its historical testimony.

This territory is grown up since the early years of the 20th century on the base of town planning projects developed especially in the period between the two world wars.

The preliminary phase of the research focused on the study of building and planning processes that led to the conformation of the observable areas today and in search of the special construction techniques. Indeed, in this urban context, constructive reality changes with respect to the canons of tradition and is a mirror of the particular historical moment,

\footnotetext{
Corresponding author: Alessio Lodato, Ph.D. student, research field: earthquake engineering. E-mail: alodato@unisa.it.
}

characterized by the advent of reinforced concrete which will lead to a new structural design of buildings. The constructions belonging to the beginning of the 20th century represent the results of an architectural knowledge, known as "transition period", in which traditional and modern skills coexist, the former related to the rule of art and the latter characterized by a copious experimental activity related to the industrial revolution [1]. In this period, architects and construction firms put great attention to the innovative techniques and materials, to the fascinating possibility of their use and to their durability.

The modernizing spirit which spread the country in those years, especially in Salerno, substantially led to the development of new experimental technologies, rather than stylistic and architectural ones [2]. Indeed, for the realization of the individual construction elements, patents and new techniques were introduced that concerned the new structural material: reinforced concrete. The work, focused on a large-scale area of study, wants to contribute to the development of a methodological process that allows expeditious analysis of static and seismic risk of buildings 
belonging to the urban centers. As known, the seismic risk assessment of an urbanized area is related to the estimation of three basic parameters: the hazard, vulnerability and exposure. The hazard represents the probability of resentments in the investigated territory of the seismic intensity set; This is a function of genetic seismic areas (areas of foliation) next to the site of interest. The vulnerability, however, describes the propensity to damage of the built and depends on the intrinsic seismic resistance characteristics of itself. In the case of historical urban fabric, as shown by the recent earthquakes that struck the country (L'Aquila, 2009 and Emilia Romagna, 2012), these skills may be particularly reduced, considering both the limited abilities of built, as it was realized (lack of knowledge and/or appropriate regulations), and the deterioration of the buildings during time (a lack of maintenance and/or improper modifications). The work views the seismic risk assessment of the historical buildings located in an urban area of the city of Salerno using three different vulnerability assessment methodologies [2] for comparison. These methods are based on statistical data and use parameters referring to the information collected in the AeDES (Feasibility and Damage in Seismic Emergency) first level form [3]: This was born as a check-list to guide engineers involved in post-earthquake inspection campaigns engaged to evaluate the feasibility of the buildings, and can be used as an instrument for collecting data and topological metrics aimed at evaluating the vulnerability of ordinary buildings.

Then, intersecting the vulnerability data with the site hazards ones, it was possible to define city damage scenarios as, with the integration of more detailed analysis and specific strategies of prevention, aiming at safety and the preservation of historical buildings.

\section{Structural Peculiarities of the Study Area}

The examined area, set against the ancient walls, was one of the first areas of expansion towards the east of the city, starting already from the early years of the 20th century. The district (Fig. 1), hub of a series of interventions and town planning projects developed in the first half of the century, can be defined as a hinge zone between the old historical center, bounded by defensive walls for centuries, and the expanded city, the centrifuge, which began to extend the urban area already in the years immediately after the Second World War. The demolition of the defensive walls in the 19th century opens the urban structure to the territory, placing the needs of programming the direction of development. Despite the first initiatives of development, however, only in the 20th century, especially in the period between the two world wars, Salerno will abandon the closed city configuration in favour of a redefinition of the urban fabric, characterized in modern sense.

The area mainly comprises the first areas of expansion result of the two land-use plans that have changed forever the image of the city of Salerno: extension plan to the sea by Engineer Franklin Colamonico, land-use plan of the new eastern district, signed by the Engineers Ernesto Donzelli and Nicola Cavaccini.

The area object of study is an historical evidence of that particular period of transition of the city, which came to a modernization that concerned the whole social and urban fabric, involving all sectors of the civil activities, both public and private. Even the construction reality of this area is a mirror of the particular historical moment. The buildings are marked by the progressive use of concrete reinforced concrete, which changes the structural concept of the building. In fact, the ancient and well-established typology of construction of load-bearing masonry with vaulted horizontal elements or isostatic (slab with beams), moved to a new "mixed" type, introducing in masonry construction reinforced concrete elements, especially floor slabs. The slab, which has become rigid and strongly connected to the box, introducing an effective system of horizontal 
chaining which eliminated the problems of eccentricity due to the asymmetry of the offsets of the walls but, above all, changed the behavior of the building under horizontal loads. The building techniques for the construction of the floor slabs were an emblematic expression of this particular historical moment: In fact, many patents and experiments were introduced. Among the typologies most used, so as to be regarded as typical of Salerno, were the floor slabs Cannovale Delle Piane: mixed slab in reinforced concrete constituted by parallel ridges, hollow tiles (called precisely Cannavali) and upper concrete slab of about $5 \mathrm{~cm}$ which is extended through the entire thickness of the perimeter walls. Other slab used in the building industry of Salerno was the slab with Zeni blocks (or Z, as she was often mentioned in the technical reports of the time), characterized by hollow tiles with a length of about $21 \mathrm{~cm}$ and commercialized with different heights. The presence of slab type Stimip and Duplex was significant [4]: The first was designed so as to achieve an air chamber in order to give the slab a high thermal insulation and was, therefore, used in roofing decks. The tiles are constituted by two lateral elements with L-form, by a perforated upper of closure and a fired tile resting on the lower wings for the formation of the ceiling. The perforated upper hollow flat block, with $5 \mathrm{~cm}$ of thickness, presents deep grooves, filled with concrete, which behave like a mixed slab; The latter, however, patented by Danusso [5], is made with reinforced concrete crossed support strips thanks to the assembly of the tile blocks such as to allow, in both orthogonal directions, the realization of joists with average thickness of $10 \mathrm{~cm}$. The tile blocks and the joists are completed with a slab in reinforced concrete with a minimum thickness of $5 \mathrm{~cm}$. The constructive framework of the vertical structures is dominated by the traditional yellow tuff masonry and, in general, for the last floors in elevation, by the gray tuff. The foundations of the buildings were made of limestone and were often supplemented with a leveling layer of brick on which the rising masonry was framed. The kind of scheme systematically used was defined as "little blocks", which was applied by early decades of the 19th century until the mid-20th century when the mechanical extraction of the tuff was introduced. The masonry technique "little blocks" employed blocks of tuff, called, according to the ratio between the sides of the outer faces, stones "by one" $(h: l=1: 1)$, "by one and a half" $(h: l=1: 1.5)$ and "two" $(h: l=1: 2)$. These blocks, with a truncated pyramid shape, squared on the outer face and notched within the interior sector, were attached to the nucleus of the rubble masonry packed with irregular stones and abundant mortar, often with low content of lime. So, the masonry is not perfectly connected within the wall thickness, despite being regular in the outer walls [6]. Most of the masonry buildings with this type of scheme were judged fairly poor from the seismic behavior point of view; while for certain buildings, the seismic behavior of the masonry has been reputed better thanks to the presence of horizontal layers of bricks, which allow a better regularity and a more effective degree of connection between the facing walls.

To characterize the buildings in this particular historical period, also, the first anti-seismic regulations were issued since the disastrous earthquake that struck Messina and Reggio Calabria in 1908. These regulations imposed limitations regarding geometry and morphology of the buildings (height, inter-storey height, distance between apertures and edges, thickness of the walls) and their context (width of the roads). As building systems, they were imposed only slum dwellers or framed with wood frame, iron, concrete or reinforced masonry, limiting the ordinary masonry, brick or stone blocks or edged, to the buildings with only ground floor.

\section{Seismic Risk Evaluation}

The study compares three procedures employed for the vulnerability analysis, based largely on data collected in AeDES sheet. These procedures are classifiable in 


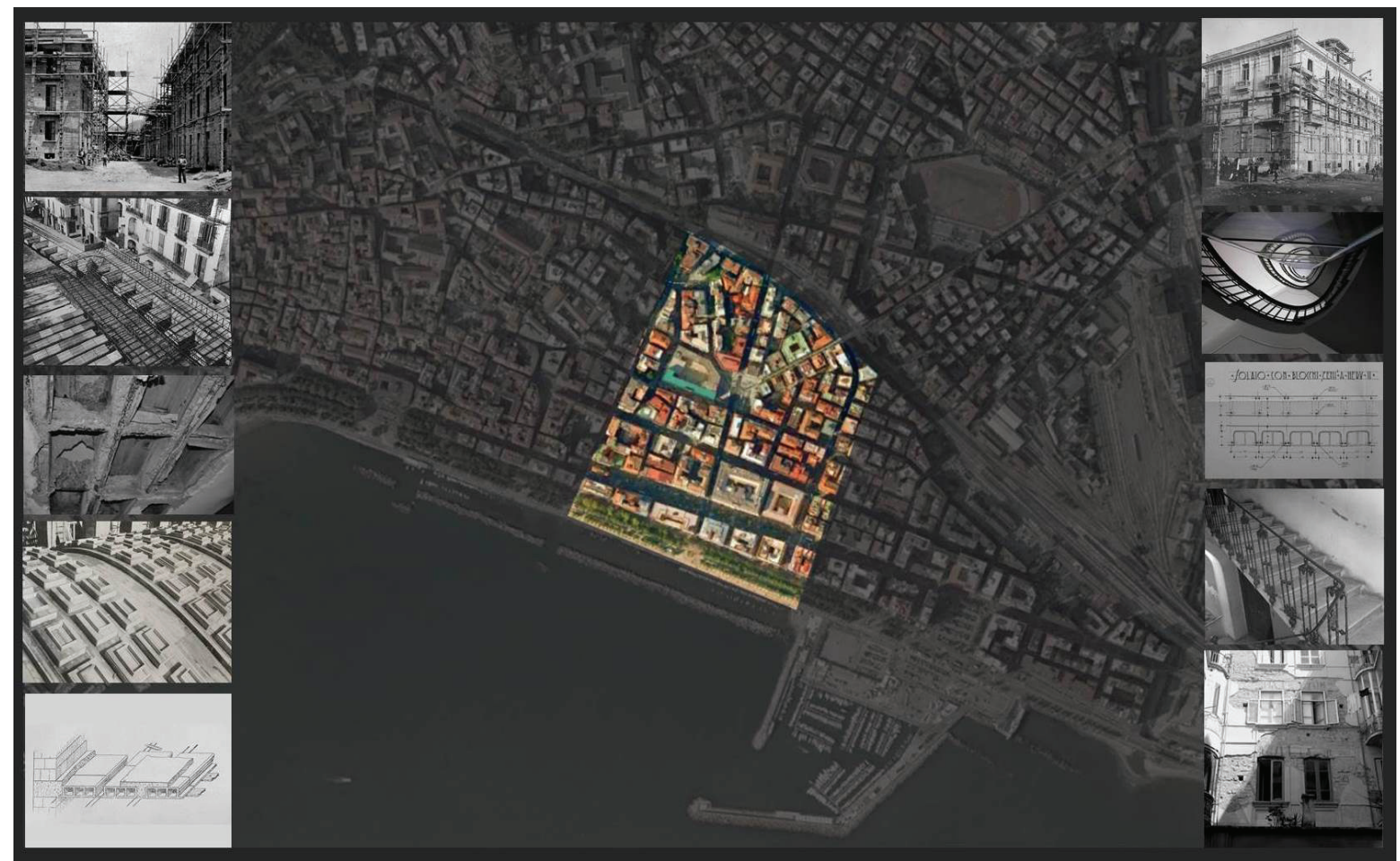

Fig. 1 Highlighting of the experimental area within the historic center of the city of Salerno. The main construction methods of the buildings analyzed in the study are shown.

the methodologies of the first level and allow to estimate the seismic resistance of ordinary buildings on the basis of observed vulnerability, as they are based on seismic severity-damage correlations obtained for predetermined structural types based on the analysis of the observed damages on buildings affected by past earthquakes.

On the basis of the results obtained by the three procedures used, it is possible to make an initial classification grouping the buildings into classes "high", "medium" and "low" of vulnerability. The seismic behavior of buildings has been defined, in particular, by classes of vulnerabilities, which can be associated to a damage probability curve, that is, a fragility curve. The first procedure considered in the work is that related to AeDES sheet, which allows to assign a vulnerability grade to individual buildings rather than a true extension of the damage, based on the indicators contained in Section 3 of AeDES sheet, considering the vertical structures-horizontal structures typology, the presence of isolated columns, the regularity in the plan and elevation, the regularity in the arrangement of infill and type of roofing by assigning appropriate weights, as shown in Eq. (1):

$$
\begin{gathered}
p_{1} \begin{cases}=0, & \text { no isolated columns } \\
=1, & \text { isolated columns }\end{cases} \\
p_{2} \begin{cases}=2, & \text { irregular structure } \\
=0, & \text { regular structure }\end{cases} \\
p_{3} \begin{cases}=2, & \text { regular facade cladding } \\
=0, & \text { irregular facade cladding }\end{cases} \\
p_{4} \begin{cases}=2, & \text { pushing heavy coverage } \\
=1, & \text { no pushing heavy coverage } \\
=3, & \text { no pushing light coverage }\end{cases}
\end{gathered}
$$

The second procedure considered is based, however, on the use of damage probability matrices DPM (damage probability matrix) described in the study by Braga et al. [7]. These matrices, description of the probability that it might have a certain level of damage considering the building typology and materials (Table 1), are the result of the analysis of damage recorded in the earthquake that struck Irpinia 
and Basilicata in 1980. In particular, the probability of having a damage $k$ (MCS (Mercalli-Cancani-Sieberg scale)) for a given level of shaking $i$ (MSK (Medvedev-Sponheuer-Karnik scale)) for the considered classes $h$ (A, B, C1, C2) of seismic vulnerability is given by the relation:

$$
P_{h k i}=\frac{5 !}{k !(5-k) !} d_{h i}^{k}\left(1-d_{h i}\right)^{5-k}
$$

where, $d_{h i}$ is described by the above-mentioned damage probability matrices.

To the scope, an appropriate correlation between the descriptive data of the vulnerability classes provided in the DPM damage probability matrices and indicators provided by the AeDES sheet has been considered (Table 1).

Finally, in the second methodology, it has been proposed to calculate the vulnerability index in an alternative way as provided by the ISTAT (National Institute of Statistics) classification [8]. In the present study, this methodology is used in order to achieve, by means of the data obtained from the AeDES sheet, the quantification of vulnerability through an index and is related to the values contained in the EMS 98 (European macroseismic scale). The AeDES sheet provides information about each building considerably larger than that deduced from ISTAT data, and thus can be used for a more comprehensive estimate of the index of vulnerability $I_{v}$ and the vulnerability class EMS 98.

The vulnerability index is calculated according to Eq. (3), in which each term of the equation is a particular numerical value associated with each metric and typological factor contained in the AeDES sheet and obtainable from ISTAT data. In particular, $I_{b}(k)$ is the base vulnerability for the type $k$, corresponding to the hypothesis of belonging to the highest age group $(i=1)$ and the most unfavorable combination of all the typological factors considered even in the ISTAT data $91(j=1)$. Instead, factors $i$ and $j$ refer, respectively, to the influence of the construction age group (more conservative) or retrofit (most optimistic) and the geometrical factors, the latter referring to the relative position of the building compared to other contiguous and to the number of floors. The term classif $(k)$ is a deduction applied to age group $i>i_{c}$, where $i_{c}$ is the age group of the seismic classification of the territory under examination. $F_{r}$ are the values for the other factors in the AeDES sheet, in particular those related to the type of roofing, pre-existing damage, morphology of the site and damage typology:

$I_{v}=I_{b}(k)+\Delta i(k) \frac{(i-1)}{5}+\Delta j(k) \frac{(j-1)}{5}+\operatorname{classif}(k)+\sum_{\mathrm{l}=1}^{4} F_{r}$

By correlating the values of $I_{v}$ with the requirements of the EMS 98 scale, it is possible to classify the built in vulnerability classes, according to the scale ranges (Class A for $I_{v}>50$, Class B for $40<$ $I_{v} \leq 50$ and Class $\mathrm{C}$ for $30<I_{v} \leq 40$ ). According to this classification, $19 \%$ of the built analyzed belongs to Class A (highest vulnerability), $70.7 \%$ to Class B and $10.3 \%$ to Class C.

The severity-damage correlation curves are trilinear curves, uniquely defined by the points $s_{i}$, the severity level of seismic activity which corresponds to the beginning of the collapse of the structure, and $s_{c}$, seismic severity of collapse of the structure. That is, according to Eq. (4):

Table 1 Correspondence between AeDES sheet data and 13 structural typologies at the base of DPM and assignment of a class of vulnerability to each of them.

\begin{tabular}{lllll}
\hline Horizontal structure & \multicolumn{4}{c}{ Vertical structure } \\
\cline { 2 - 5 } & Poor masonry (B-C) & Midding masonry (D) $^{*}$ & Good masonry (E) & Reinforced concrete $^{*}$ \\
\hline${\text { Vaults }(2-3)^{*}}^{*}$ & A & A & A & - \\
Deformable floors (4) & A & A & C1 & - \\
Semi-rigid floors (5) & B & B & C1 & - \\
Rigid floors (6) & B & C1 & C1 & C2 \\
\hline
\end{tabular}

Note: ${ }^{*}$ Refers to AeDES sheet classification. 


$$
d(s)= \begin{cases}d=0 & \text { for } s<s_{i} \\ d=\frac{s-s_{i}}{s_{c}-s_{i}} & \text { for } s_{i}<s<s_{c} \\ d=1 & \text { for } s \geq s_{c}\end{cases}
$$

Differently from the first method, which provides a direct correlation between severity and damage through DPM, the second methodology is appropriate to establish such relations function of the vulnerability index $I_{v}$.

To this end, we have the following empirical relationships [1]:

$$
\begin{aligned}
& P G A_{i}=\alpha_{i} e^{\left(-\beta_{i} I_{v}\right)} \\
& P G A_{c}=\left(\alpha_{c}+\beta_{c} I_{v}^{\gamma}\right)^{-1}
\end{aligned}
$$

where, $\alpha_{i}=0.08, \beta_{i}=0.0195, \alpha_{c}=1.53, \beta_{c}=0.00191$, $\gamma=1.8$, which define the initial collapse acceleration $\left(P G A_{i}\right)$ and the collapse acceleration $\left(P G A_{c}\right)$ of the structure, having fixed the vulnerability index $I_{v}$.

\section{Risk Assessment and Damage Scenarios}

Another decisive step for the unitary seismic risk analysis is the assessment of seismic hazard. The starting point is the seismic hazard, derivable from hazard maps, developed by INGV (National Institute of Geophysics and Volcanology), which provide the PGA (peak ground acceleration) in a given reference period, $V_{r}=V_{n} \cdot C_{u}$. In the present study, the reference accelerations corresponding to the four limit states provided by the actual Italian code [9] (SLO: operational limit state; $S L D$ : damage limit state; $S L V$ : life safety limit state; $S L C$ : collapse prevention limit state) and reference periods, respectively of 50,75 and 100 years, have been considered, since, according to the classification of OPCM 3274 of 2003, in the district under examination, there is the presence of ordinary buildings, relevant buildings and strategic buildings. Studies on local seismic hazard were possible to estimate the local peak ground accelerations (referred to $P G A^{*}$ ) according to Eq. (6), in accordance with the provisions of the regulations:

$$
P G A^{*}=P G A \cdot S s \cdot S t
$$

where, $S s$ and $S t$ are, respectively, the stratigraphic amplification coefficient and the topographic amplification coefficient of the site. The accelerations framework considered in the district (characterized by two different values of $S$ and, therefore, divided into two sub zones) is shown in Table 2.

Finally, correlating the results obtained from the vulnerability analysis with those related to the site seismic hazard, it was possible to obtain severity-damage curves representative of the seismic behavior of historical buildings analyzed and achieve the expected damage scenarios [10]. The results, summarized in Fig. 2, show the expected damage of historical buildings in the area object of the study for

\begin{tabular}{|c|c|c|c|c|}
\hline \multicolumn{5}{|c|}{$P G A^{*}\left(9,822 \mathrm{~m} / \mathrm{s}^{2}\right)$ for ordinary buildings $(V r=50$ years $)$} \\
\hline Zone/limit state & SLO & $S L D$ & $S L V$ & $S L C$ \\
\hline Zone $1(S=1.2)$ & 0.030 & 0.060 & 0.15 & 0.18 \\
\hline Zone $2(S=1.3)$ & 0.033 & 0.065 & 0.16 & 0.20 \\
\hline \multicolumn{5}{|c|}{$P G A^{*}\left(9,822 \mathrm{~m} / \mathrm{s}^{2}\right)$ for relevant buildings $(V r=75$ years $)$} \\
\hline Zone/limit state & SLO & $S L D$ & $S L V$ & $S L C$ \\
\hline Zone $1(S=1.2)$ & 0.06 & 0.09 & 0.15 & 0.18 \\
\hline Zone $2(S=1.3)$ & 0.065 & 0.10 & 0.20 & 0.20 \\
\hline \multicolumn{5}{|c|}{$P G A^{*}\left(9,822 \mathrm{~m} / \mathrm{s}^{2}\right)$ for strategic buildings $(V r=100$ years $)$} \\
\hline Zone/limit state & $S L O$ & $S L D$ & $S L V$ & $S L C$ \\
\hline Zone $1(S=1.2)$ & 0.09 & 0.09 & 0.18 & 0.21 \\
\hline Zone $2(S=1.3)$ & 0.098 & 0.098 & 0.20 & 0.23 \\
\hline
\end{tabular}
different limit states corresponding to different level of seismic excitation.

Table 2 Peak ground acceleration expected in the two sub-zones of the experimental area. 

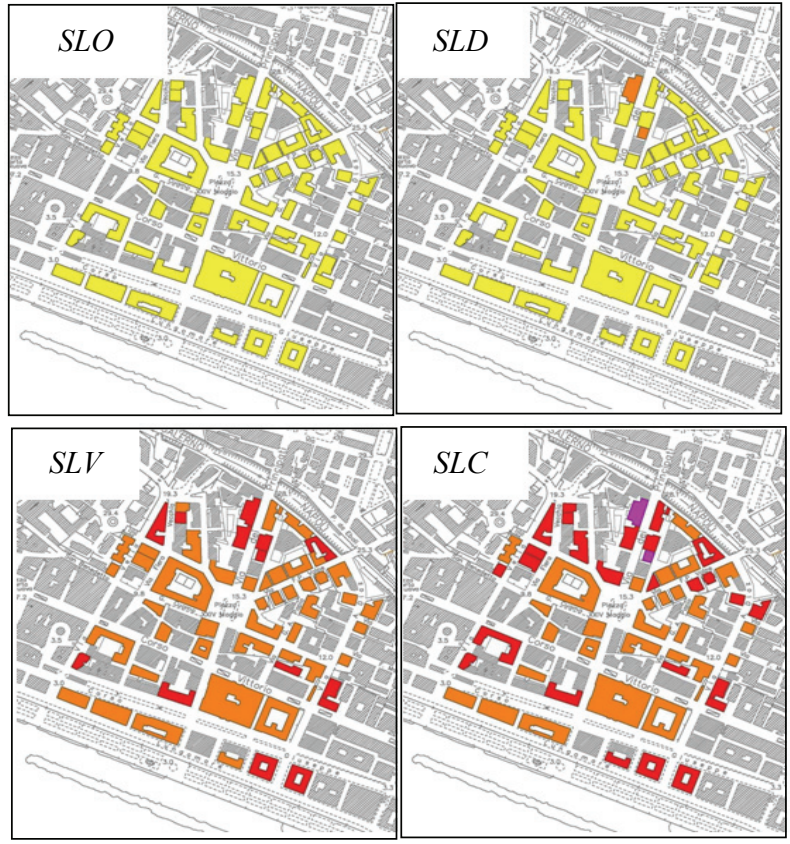

Damage levels:

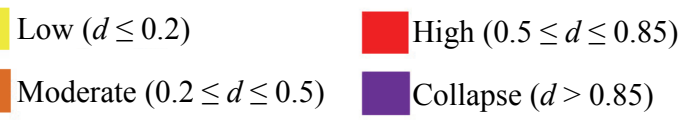

Fig. 2 Expected damage scenarios in the area of interest.

\section{Conclusions}

The study showed that, although the territory of Salerno being characterized by telluric events expected potentially non violent compared to other realities of our national territory, the condition of the buildings, however, can lead, in the case of earthquakes with a high return period, to widespread and severe damage, and sometimes the collapse of the structures which are most vulnerable.

The proposed results are preliminary, they provide an estimate of the order of magnitude related to the assessment of seismic risk in large scale for the architectural and historical heritage analyzed. Despite its specificity, this study aims to highlight the issues related to the safety and preservation of established urban centers and the procedures content in it allow to have relevant effects on the land use planning policies and infrastructure management [11].

The survey of the levels of pre-existing risk within the historical settlements, areas most seismically vulnerable in the cities, should aim to define the seismic functionality of the entire urban system. The response of a city or a territory at one natural event comes not only from the sum of the vulnerability of the buildings, but especially by the set of interrelationships that are established between the different roles and parts of the city.

Our country is characterized by a medium seismicity, but also by a very high vulnerability, considering even the high presence of historical buildings, built without any earthquake engineering prescription. A potential collapse of these buildings means huge loss not only in terms of human lives but also in terms of cultural and historical values.

It follows that the decisive step in order to mitigate both the time of the assessment of the seismic risk of the established centers, which must begin with an analysis of large scale [12]. In case of seismic event, this step can speed up the assistance to the affected population, the recovery of the affected areas, quick re-functionality of the urban constructions (homes, offices, infrastructure), the resumption of economic activities, social and cultural relations and memory characteristics of historical settlements.

\section{References}

[1] Croce, A., Tessitore, F., and Conte, D. 2006. Naples and Campania in the Twentieth Century. Diary of a Century. Vol. II. Napoli: Liguori Editore. (in Italian)

[2] Ribera, F., and Dolgetta, P. 2010. "School Buildings in Salerno between the Wars: The Evolution of the Way of Building between Tradition and Modernity." In History of Engineering. Vol. 1. Napoli: Cuzzolin, 543-55. (in Italian)

[3] Baggio, C., Bernardini, A., Colozza, R., Corazza, L., Bella, M. D., Di Pasquale, G., Dolce, M., Goretti, A., Martinelli, A., Orsini, G., Papa, F., and Zuccaro, G. 2009. Manual for the Compilation of the I Level Damage Detection Sheet, Emergency and Feasibility for Ordinary Buildings in the Post-earthquake Emergency (AeDES). Dipartimento della Protezione Civile (Department of Civil Protection). (in Italian)

[4] Miccio, G., and Ribera, F. 2010. "Building Development and Construction Techniques in Salerno between the Wars." In The Town Hall, edited by Zampino, G., and 
Salerno, F. M. Napoli: Paparo Edition, 97-115. (in Italian)

[5] Danusso, A. 1912. Duplex: Slab in Reinforced Concrete, Double Order Beams, Inner Tube, Plan Ceiling. Milano: Stabilimento Lito-Tipografico Antonio Macciachini. (in Italian)

[6] De Sivo, B., and Iovino, R. 1996. Manual Recovery of Ancient Building Techniques of Naples from the Fourteenth to the Nineteenth Century. Napoli: CLEAN.

[7] Braga, F., Dolce, M., and Liberatore, D. 1982. "A Statistical Study on Damaged Buildings and Ensuing Review of the MSK-76 Scale." Presented at the 7th European Conference on Earthquake Engineering, Athens, Greece.

[8] Bernardini, A., Biscontin, I., and Scattolin, M. 2005. Vulnerability and Damage Scenarios of Ordinary Buildings in the Mansfield Town (TV). A technical report in Research Project 2000-2002: Scenarios of Damage in the Veneto-Friuli Area. (in Italian)

[9] Italian Ministry of Infrastructure. 2008. Italian Technical Code for Buildings. D.M. Infrastructure 14 January 2008. Italian Ministry of Infrastructure. (in Italian)

[10] Gaugenti, E., and Petrini, V. 1989. "The Case of Old Buildings: Towards a New Law Damage-Intensity." In Proceedings of the 4th National Conference on Earthquake Engineering, 145-53. (in Italian)

[11] Marino, I., Petti, L., and Palazzo, B. 2010. "A Large-Scale Strategy for Seismic Risk Mitigation of Infrastructure: The Case of Campania Region." Ingegneria Sismica 2: 34-45. (in Italian)

[12] Palazzo, B., Petti, L., and Albano, G. 2001. "Optimal Strategies for Seismic Risk Reduction.” Presented at the Third International Conference on Earthquake Resistant Engineering Structures, Malaga, Spain. 